\title{
dc Conductivity of an array of Josephson junctions in the insulating state
}

\author{
S. V. Syzranov ${ }^{1}$, K. B. Efetov ${ }^{1}$, and B. L. Altshuler ${ }^{2}$ \\ ${ }^{1}$ Theoretische Physik III, Ruhr-Universität Bochum, D-44801 Bochum, Germany \\ ${ }^{2}$ Physics Department, Columbia University, New York, N.Y. 10027, USA
}

(Dated: November 17, 2018)

\begin{abstract}
We consider microscopically low-temperature transport in weakly disordered arrays of Josephson junctions in the Coulomb blockade regime. We demonstrate that at sufficiently low temperatures the main contribution to the dc conductivity comes from the motion of single-Cooper-pair excitations, scattered by irregularities in the array. Being proportional to the concentration of the excitations, the conductivity is exponentially small in temperature with the activation energy close to the charging energy of a Cooper pair on a superconductive island. Applying a diagrammatic approach to treat the disorder potential we calculate the Drude-like conductivity and obtain weak localization corrections. At sufficiently low temperatures or strong disorder the Anderson localization of Cooper pairs ensues.

PACS numbers: 74.81.Fa, 71.30.+h, 73.23.Hk, 74.50.+r
\end{abstract}

Artificially fabricated Josephson junction arrays (JJA) reveal various fundamental quantum phenomena ranging from quantum phase transitions to the motion of single charges and vortices (see, e.g. [1]). The flexibility of their design and parameters makes JJAs a perfect laboratory for study of physics underlying these phenomena.

Low-temperature transport in the array is determined by the ratio of the characteristic Josephson coupling energy $J$ to the effective charging energy $B / 2$ of adding one Cooper pair on a superconductive island in the array. In the two limiting cases, $J>J_{c}$ and $J<J_{c}$, where $J_{c} \sim B$, the JJA is known to be macroscopically superconducting or insulating, respectively, which has been demonstrated by a microscopic calculation quite long ago [2]. Especially interesting is the two-dimensional $(2 D)$ case when a Josephson- or charging-energy- dominated array may undergo respectively a vortex- or charge- unbinding Berezinskii-Kosterlitz-Thouless (BKT) [3] transition to a normal conducting state.

Extensive studies of JJA dynamics have been carried out since the first array fabrication [4]. However, transport properties of the Coulomb-blockaded JJAs are not fully understood yet, although the issue of the conductivity of a granulated superconductor in the insulating phase has first been addressed already in Ref. [2]. It was demonstrated that the frequency-dependent conductivity $\sigma(\omega)$ had sharp peaks at frequencies corresponding to the excitation energies of Cooper pairs. However, the $d c$ conductivity in the insulating state has not been calculated explicitly.

Later, the conductivity of large JJAs has been studied close to the superconductor-insulator transition using mean-field-type approaches [5] or scaling arguments based on the charge-vortex duality [6]. In many respects the study of transport was phenomenological and, particularly, did not account properly for the effects of disorder.

However, as the relaxation of charge carrying excitations due to inelastic processes strongly decreases with temperature, the disorder must play the major role in the low-temperature transport in the insulating phase of the array.

Actually, disorder is intrinsically present in the conventional tunnelling Hamiltonian describing the coupling between superconducting islands, as electrons can hop from one island to any state near the Fermi surface in another island. Such a disorder determines the conductivity of a regular array of normal metal grains (see, e.g., Ref. 7]). Is the same true also for the insulating state of a regular array of superconducting islands or the macroscopic irregularities in the system should be accounted for? How does the conductivity depend on the temperature and on the range of electron-electron interactions?

In the present Letter we study a large JJA deeply in the insulating state $(J \ll B / 2)$ and address these questions. Most of our results are valid for any dimensionality of the array, although at some points we restrict ourselves to $2 D$. We assume for simplicity that the superconducting gap $\Delta$ in a single island is the largest energy scale.

We calculate the conductivity of the JJA under rather general assumptions and demonstrate that the conductivity of a regular periodic array without a macroscopic disorder remains infinitely large as long as macroscopic disorder and inelastic processes are neglected. The $d c$ is carried mainly by single-Cooper-pair excitations with the charge $\pm 2 e$. Such bosonic particles move in a regular array without being scattered. At the same time, the density of the bosons is exponentially low in temperature, $\propto \exp \left(-E_{0} / T\right)$, with $E_{0}$ close to $B / 2$.

Macroscopic disorder in the JJA results in the boson scattering on irregularities and makes the conductivity finite. The conductivity is proportional to the density of the bosons, i.e to $\exp \left(-E_{0} / T\right)$, in the limit $T \ll E_{d}$

$$
\sigma \sim e^{2} T^{-1} \tau(\min (T, J))^{2} \exp \left(-E_{0} / T\right),
$$

where $\tau$ is the elastic scattering time and $E_{d}$ is the energy of two bosons of opposite charge (boson dipole) located on neighboring islands. The energy $E_{d}$ is either of the same order as $E_{0}$ or considerably smaller depending on 
the range of the effective Coulomb interaction between the bosons, determined by the capacitive properties of the array. Eq. (1) is an analogue of the classical expression for the conductivity of free particles. In the limit $T \ll J$ the pre-exponential can be evaluated exactly and equals $4 e^{2} T \tau / \pi$.

Remarkably, the description in terms of scattered bosons allows one not only to obtain the classical limit, Eq. (1), but also to describe the quantum interference leading to localization effects. In $2 D$ the first weak localization correction $\delta \sigma_{W L}$ to $\sigma$ takes the form

$$
\delta \sigma_{W L} \sim-e^{2} T^{-1} \min (J, T) \exp \left(-E_{0} / T\right) \ln \left(L_{\phi} / l\right),
$$

where $l \sim(J \min (J, T))^{\frac{1}{2}} \tau$ is the mean free path (measured in lattice periods), and $L_{\phi}$ is the Cooper-pair dephasing length determined by their recombination, interaction with phonons, emission of Cooper-pair dipoles, etc. In the present paper we assume that the temperature is low enough $\left(T \ll E_{d}\right)$ and thus $L_{\phi} \gg l$. At $T \ll J$ the pre-exponential factor in Eq. (2) equals $-4 e^{2} / \pi^{2}$. At low temperatures or strong disorder the correction $\delta \sigma_{W L}$ becomes comparable with $\sigma$, which corresponds to the strong Anderson localization of the bosons.

Eqs. (11) and (2) are the main results of our paper. The conductivity of a JJA in the insulating phase is similar to that for electrons in disordered metals but contains in addition the activation exponent determining the density of the excited Cooper pairs.

Now we formulate the model and derive the above results. We start with a standard effective Hamiltonian $\hat{\mathcal{H}}$ [2] describing the motion of Cooper pairs in a JJA

$$
\hat{\mathcal{H}}=\frac{1}{2} \sum_{i, j} B_{i j} \hat{n}_{i} \hat{n}_{j}-\sum_{i, j} J_{i j} \cos \left(\phi_{i}-\phi_{j}\right),
$$

where the indices $i$ and $j$ label the superconducting islands, $B_{i j}$ is the inverse capacitance matrix of the array in units of $(2 e)^{2}, \phi_{i}$ and $\hat{n}_{i}=-i \partial / \partial \phi_{i}$ are respectively the phase of the superconducting order parameter and the operator of the number of excess Cooper pairs in the island $i . J_{i j}$ is the energy of Josephson coupling between neighboring islands $i$ and $j, J_{i j}=J_{j i}=J$. In these notations, adding a Cooper pair to the site $i$ requires the charging energy $B_{i i} / 2$, which is site-dependent. The average value of this energy is $B / 2 \equiv\left\langle B_{i i} / 2\right\rangle$. We consider the JJA deeply in the insulating phase, $J \ll B$.

Irregularities of the array can be described by the fluctuations $\delta B_{i j}$ and $\delta J_{i j}$. As we show below, current in the array is carried by the individual bosons or antibosons describing respectively excess Cooper pairs on the islands or "Cooper-pair-holes". Random offset charges weakly coupled to the array may shift the energy of a Cooper pair on an island, and, thus, contribute to the fluctuations of the coefficients $B_{i i}$.

Neglecting the Josephson couplings and disorder one obtains a discrete spectrum of the excitation energies of the system determined by the eigenvalues of the first term in Eq. (33). The eigenvalues of the operators $\hat{n}_{i}$ are integers. The ground state corresponds to all $n_{i}=0$. All excited states are degenerate as long as the system remains translationally invariant. Of course, no $d c$ current can flow through the system in this limit.

The degeneracy of the excited states is lifted in the presence of the Josephson tunnelling $J_{i j}$ between the islands. The Hamiltonian, Eq. (3), is equivalent to a tightbinding model for bosons: their states form a band with a width proportional to $J$. As a result, macroscopic $d c$ transport is possible. The disorder results in the scattering of the bosons inside the bands and leads to the finite conductivity, Eqs. (11) and (21).

At low temperatures the $d c$ current is carried by bosons with the charge $2 e$ and antibosons with the charge $-2 e$. The conductivity is dominated by the lowest energy bands of bosonic and antibosonic states. These two bands are located near the energy $B / 2[\underline{8}]$.

In order to calculate the conductivity at low temperatures we may thus consider a reduced Hilbert space: the $i$-th island has only three quantum states: $|0\rangle_{i}$, zero excess Cooper pairs on it, and $| \pm 1\rangle_{i}$, one Cooper pair (antipair). It is convenient to rewrite the Hamiltonian (3) in this space in terms of pseudospin operators $\hat{S}_{i}^{+}, \hat{S}_{i}^{-}$and $\hat{S}_{i}^{z}: \hat{S}_{i}^{ \pm}|0\rangle_{i}=\sqrt{2}| \pm 1\rangle_{i}, \hat{S}_{i}^{ \pm}|\mp 1\rangle_{i}=\sqrt{2}|0\rangle_{i}, \hat{S}_{i}^{ \pm}| \pm 1\rangle_{i}=0$, $\hat{S}_{i}^{z}|0\rangle_{i}=0, \hat{S}_{i}^{z}| \pm 1\rangle_{i}= \pm| \pm 1\rangle_{i}$ corresponding to the pseudospin $S_{i}=1$. The reduced Hamiltonian

$$
\hat{\mathcal{H}}_{\text {red }}=\frac{1}{2} \sum_{i, j} B_{i j} \hat{S}_{i}^{z} \hat{S}_{j}^{z}-\frac{1}{2} \sum_{i, j} J_{i j} \hat{S}_{i}^{+} \hat{S}_{j}^{-}
$$

is equivalent to an anisotropic Heisenberg spin-1 model. The pseudospin operators obey the conventional commutation relations

$$
\left[\hat{S}_{i}^{+}, \hat{S}_{j}^{-}\right]=2 \delta_{i j} \hat{S}_{i}^{z}, \quad\left[\hat{S}_{i}^{z}, \hat{S}_{j}^{ \pm}\right]= \pm \delta_{i j} \hat{S}_{i}^{ \pm} .
$$

We assume for simplicity that the islands in the array form a square lattice and begin with calculating the excitation spectrum in an ideal JJA without disorder. The states of the Hamiltonian $\hat{\mathcal{H}}_{\text {red }}$ can be classified by the $S^{z}$-projection of the total spin. The ground state corresponds to $S_{i}^{z}=0$ for all $i$.

In order to calculate the conductivity we consider states corresponding to a single boson or antiboson in the array $\left(S^{z}= \pm 1\right)$. For these states the eigenenergy of the first term of the Hamiltonian $\hat{\mathcal{H}}_{\text {red }}$, Eq. (4), equals $B / 2$. In the limit $J \ll B$, we approximate the eigenfunction of $\hat{\mathcal{H}}_{\text {red }}$ for $S^{z}= \pm 1$ by a plane wave

$$
|\mathbf{k}\rangle=N^{-\frac{1}{2}} \sum_{\mathbf{r}} e^{i \mathbf{k r}}|\mathbf{r}\rangle,
$$

where $N$ is the number of the islands in the array. The corresponding excitation spectrum takes the form

$$
E(\mathbf{k})=B / 2-2 J \cos k_{x}-2 J \cos k_{y},
$$


where $\mathbf{k}=\left(k_{x}, k_{y}\right)$. Thus, the excitation spectrum has a narrow band of the width $8 J$ separated from the ground state by the gap

$$
E_{0}=B / 2-4 J
$$

As long as the gap significantly exceeds the temperature the density of the bosons is exponentially small. In this limit the interaction between them can be neglected and we can describe the system in terms of a single-particle tight-binding Hamiltonian with the spectrum given by Eq. (7). Disoder manifests itself in the model through the fluctuating parts $\delta B$ and $\delta J$ of the parameters $B$ and $J$.

The conductance of the array can be calculated using the standard Kubo linear-response theory. The operator $\hat{I}_{i j}$ of the current between the $i$-th and $j$-th islands reads

$$
\hat{I}_{i j}=i e J_{i j}\left(\hat{S}_{j}^{+} \hat{S}_{i}^{-}-\hat{S}_{i}^{+} \hat{S}_{j}^{-}\right),
$$

Its expectation value $I_{i j}(\omega)$ can be expressed through the retarded correlation function of currents $\Pi_{i j, k l}(\omega)$ :

$$
\begin{gathered}
\Pi_{i j, k l}(\omega)=\left.\frac{1}{2} \int_{-\beta}^{\beta}\left\langle\hat{I}_{i j}(\tau) \hat{I}_{k l}(0)\right\rangle e^{i \Omega_{n} \tau} d \tau\right|_{i \Omega_{n} \rightarrow \omega+i 0}, \\
I_{i j}(\omega)=-i \omega^{-1} \sum_{(k l)}\left(\Pi_{i j, k l}(\omega)-\Pi_{i j, k l}(0)\right)\left(\mathbf{E l}_{k l}\right),
\end{gathered}
$$

where $\mathbf{E}$ is the electric field, $\mathbf{l}_{k l}$ - vector connecting islands $k$ and $l$.

Depending on whether the length $L_{x}$ of the array is smaller or larger than the mean free path $l$, the transport in the sample is respectively ballistic or diffusive.

Using Eqs. (10) and (11) in the ballistic limit we find the conductance of an $L_{x} \times L_{y}$ rectangular array

$$
G=\frac{8 e^{2}}{\pi} L_{y} \sinh (2 J / T) I_{0}(2 J / T) \exp \left(-E_{0} / T\right) .
$$

Here $I_{0}$ is the modified Bessel function. Of course, at $l \sim$ $L_{x}$ Eq. (12) matches the diffusive conductance $\sigma L_{y} / L_{x}$ with $\sigma$ given by Eq. (11).

Let us note now that in the low-temperature limit the array resembles a conventional semiconductor. Indeed, at low density of the bosons the particle statistics is not important. The bosons thus can be considered as doubly charged electrons thermally activated to the conduction band of the width $8 J$, the latter being separated from the valence band of the semiconductor by the gap $E_{0}$, Eq. (8). Hence, one can evaluate the conductivity in the diffusive regime using the standard diagrammatic technique [9]. We assume that the disorder is weak enough for the elastic scattering time $\tau$ to exceed the inverse characteristic kinetic energy $\min (J, T)$ of the bosons in the conduction band:

$$
\tau(\min (J, T)) \gg 1 .
$$

To average over the disorder we assume for simplicity a Gaussian distribution for deviations $\delta B_{i i}$ and $\delta J_{i j}$ from the average values $B$ and $J$ with correlations

$$
\left\langle\delta B_{i i} \delta B_{j j}\right\rangle=f_{1} \delta_{i j}, \quad\left\langle\delta J_{i j} \delta J_{k l}\right\rangle=f_{2}\left(\delta_{i k} \delta_{j l}+\delta_{i l} \delta_{j k}\right) .
$$

Our results Eqs. (1) and (2) with appropriate $\tau$ apply nevertheless for arbitrary not short-correlated distributions of the fluctuations.

The basic element of the perturbation theory is the contraction rule for the effective disorder potential $\hat{V}$

$$
\begin{aligned}
\left\langle\hat{V}_{\mathbf{k}_{1} \mathbf{p}_{1}} \hat{V}_{\mathbf{k}_{2} \mathbf{p}_{2}}\right\rangle= & (2 \pi)^{2} \gamma\left(\mathbf{k}_{1}+\mathbf{k}_{2}, \mathbf{k}_{1}-\mathbf{p}_{2}\right) \\
& \delta\left(\mathbf{k}_{1}+\mathbf{k}_{2}-\mathbf{p}_{1}-\mathbf{p}_{2}\right), \\
\gamma\left(\mathbf{k}_{+}, \mathbf{k}_{-}\right)= & f_{1} / 4+f_{2} \sum_{i}\left(e^{\mathbf{l}_{i} \mathbf{k}_{+}}+e^{\mathbf{l}_{i} \mathbf{k}_{-}}\right),
\end{aligned}
$$

where $\mathbf{l}_{i}(i=1 \ldots 4)$ are the unit vectors connecting an island with its nearest neighbors.

The standard procedure (see e.g. Ref. [9]) of evaluation of $\tau$ in the limit $k \ll 1$ under the condition (13) gives

$$
\tau^{-1}=\left(f_{1} / 8+4 f_{2}\right) / J .
$$

The scattering time $\tau$ remains of the same order of magnitude at arbitrary momentum $k \sim 1$.

We use the Kubo-Greenwood formula

$$
\begin{aligned}
\sigma_{\alpha \beta}= & \frac{2(2 e)^{2}}{\omega} \int \frac{d \mathbf{p}}{(2 \pi)^{2}} \int \frac{d \varepsilon}{2 \pi}(n(\varepsilon)-n(\varepsilon+\omega)) \\
& \left\langle v_{\alpha} G^{A}(\mathbf{p}, \varepsilon) v_{\beta} G^{R}(\mathbf{p}, \varepsilon)\right\rangle,
\end{aligned}
$$

where $\mathbf{v}=2 J\left(\sin k_{x}, \sin k_{y}\right)$ is the velocity of the boson, $n(\varepsilon) \approx \exp (-\varepsilon / T)$ is the Boltzman distribution of the excitations in the conduction band and $G^{R, A}(\mathbf{k}, \varepsilon)$ are retarded (advanced) Green's functions of non-interacting bosons, $\left\langle G^{R, A}(\mathbf{k}, \varepsilon)\right\rangle=(\varepsilon-E(\mathbf{k}) \pm i /(2 \tau))^{-1}$. Using the condition (13) we come to Eq. (11).

We emphasize that Eq. (1) has been obtained in the standard scheme neglecting diagrams with crossing impurity lines [9]. In disordered metals this approach is applicable in the limit $\varepsilon_{F} \tau \gg 1$, where $\varepsilon_{F}$ is the Fermi energy. Here the role of large parameter is played by $\tau(\min (J, T))[$ cf. Eq. (13)]

Next we calculate the weak localization correction $\delta \sigma_{W L}$ to the conductivity. Again, the condition (13) allows us to repeat the summation of the diagrams of Ref. [10] and to arrive at Eq. (21).

The limiting case $C / C_{0} \gg 1$, where $C$ is the mutual capacitance of neighboring islands and $C_{0}$ is the selfcapacitance, often corresponds to the experimental situation (see, e.g., Ref. [12]) and is especially interesting from the theoretical point of view. In this case the charging energy $E_{0}$ of a single boson significantly exceeds the dipole energy $E_{d}, E_{0} / E_{d} \sim \ln \left(C / C_{0}\right) \gg 1$.

Such bosons resemble vortices in superconductors [13]. A finite ratio $C / C_{0}$ determines the scale of the interaction 
of the bosons and plays the same role as the penetration depth cutting the logarithmic interaction of vortices in superconductors. In the limit $C / C_{0} \rightarrow \infty$ the energy of the bosons logarithmically diverges with the size $L$ of the sample. Using the analogy with the vortices one can expect in this limit the BKT transition, Ref. [3], with the critical temperature $T_{K}$ of order of the dipole energy $E_{d}$.

Properties of the system of the bosons are similar to those of the system of vortices in conventional $2 D$ superconductors. In the BKT scenario single vortices do not enter the system below $T_{K}$ and the system is a superfluid. In the JJA considered here, there are no single bosons in the limit $C / C_{0} \rightarrow \infty$ and the conductivity vanishes. However, the BKT transition in superconductors is known to smear because the energy of a single vortex is finite due to the finite penetration depth, which makes vortices itinerant resulting in a finite resistivity. In the JJA the energy of the bosons is finite due to the finite $C / C_{0}$ and their motion makes the electric transport possible. As the density of the bosons is proportional to $\exp \left(-E_{0} / T\right)$, so is the conductivity, Eq. (11). At temperatures $T \ll E_{d} \sim T_{K}$ one can neglect the presence of dipole excitations in the array which could lead to an additional scattering or relaxation of the single bosons and to a screening of Coulomb interaction.

At $T>T_{K}$ single vortices in 2D superconductors exist due to the entropy contribution, Ref. [3], and the superfluidity is destroyed. 2D superconductors, strictly speaking, do not exist. Thus, as the superfluidity below $T_{K}$ is either absent, one obtains a crossover from the finite exponentially small resistivity contributed by the motion of single vortices to the resistivity of normal metals. Analogously, in a JJA one can expect a crossover from the exponentially low conductivity at $T \ll E_{d}$, Eq. (1), to a temperature-independent conductivity at $T>E_{d}$. In other words, the Coulomb blockade effects are important at low temperature $T<E_{d}$ but can be neglected at $T>E_{d}$. Interference of the bosons results in the existence of one more temperature region where effects of localization can play an important role and the Coulomb blockade is further enforced by the Anderson localization.

Recently, a similar model of a $2 D$ JJA (but without disorder) has been suggested in Refs. [14] and [15] to describe the experiments on strongly disordered superconductors [15, 16]. The authors obtained an exponential behavior of the conductivity with the activation gap $B / 2$ for $T>E_{d}$ and a double exponential behavior for $T \ll E_{d}$. Clearly, our results, Eqs. (1), (2), and the absence of the Coulomb blockade at $T>E_{d}$ are in a strong disagreement with those findings. A detailed criticism of the theoretical treatment of Refs. [14 and [15] can be found in Ref. 17]. A comparison of our results with the results of relevant experimental works will be presented elsewhere. Also, it would be interesting to clarify the question of the many-body localization [11] of the bosons in the system under consideration.
In conclusion, we calculated the $d c$ conductivity of a large Coulomb-blockaded Josephson junction array at low temperatures and demonstrated that it is determined by the thermally activated single-Cooper-pair excitations on the superconducting islands. In the absence of macroscopic structural disorder in the array the transport is ballistic. In the presence of sufficiently weak disorder the conductivity in a certain temperature range is described by the Drude-type formula multiplied by the activation exponent $\exp \left(-E_{0} / T\right)$, where $E_{0}$ is the Coulomb energy of the single-Cooper-pair excitation. Lowering the temperature below some characteristic value results in even faster decrease of the conductivity due to the Anderson localization of Cooper pairs.

The authors thank M. Yu. Kharitonov for discussions. The work has been financially supported by SFB Transregio 12, SFB 491, and US DOE contract No. DE-AC02$06 \mathrm{CH} 11357$.

[1] R. Fazio and H. van der Zant, Phys. Rep. 355, 235 (2001).

[2] K. B. Efetov, Zh. Eksp. Teor. Fiz. 78, 2017 (1980) [Sov. Phys. JETP 51, 1015 (1980)].

[3] V. L. Berezinskii, Zh. Eksp. Teor. Fiz. 59, 907 (1970) [Sov. Phys. JETP 32, 493 (1971)]; J. M. Kosterlitz and D. J. Thouless, J. Phys. C6, 1181 (1973).

[4] R. F. Voss and R. A. Webb, Phys. Rev. B 25, 3446 (1982); R. A. Webb et al., Phys. Rev. Lett. 51, 690 (1983).

[5] C. Bruder et al., Phys. Scr. t42, 159 (1992); A. van Otterlo et al., Phys. Rev. B 48, 3316 (1993).

[6] M. P. A. Fisher, Phys. Rev. Lett. 65, 923 (1990); M. P. A. Fisher, G. Grinstein, and S. M. Girvin, Phys. Rev. Lett. 64, 587 (1990).

[7] I. S. Beloborodov, A. V. Lopatin, V. M. Vinokur, and K. B. Efetov, Rev. Mod. Phys. 79, 469 (2007).

[8] Note, in analogy with semiconductors there can also be neutral excitations with energies below $B / 2$ (excitons). However, they have a little effect on the conductivity.

[9] A. A. Abrikosov, L. P. Gorkov, and I. E. Dzyaloshinski, Methods of Quantum Field Theory in Statistical Physics (Dover, New York, 1975).

[10] L. P. Gorkov, A. I. Larkin, and D. E. Khmelnitskii, Pis'ma v ZhETF 30, 248 (1979) [JETP Lett. 30, 228 (1979)].

[11] D. M. Basko, I. L. Aleiner, and B. L. Altshuler, Ann. Phys. 321, 1126 (2006).

[12] J. E. Mooij et al., Phys. Rev. Lett. 65, 645 (1990).

[13] P.G. De Gennes, Superconductivity of Metals and Alloys (Benjamin, New York, 1966).

[14] M. V. Fistul, V. M. Vinokur, and T. I. Baturina, Phys. Rev. Lett. 100, 086805 (2008).

[15] V. M. Vinokur et al., Nature 452, 613 (2008).

[16] G. Sambandamurthy et al., Phys. Rev. Lett. 94, 017003 (2005).

[17] K. B. Efetov, M. V. Feigel'man, and P. B. Wiegmann, arXiv e-prints (2008), 0804.3775, Phys. Rev. Lett. 102, 049701 (2009). 